\title{
LIGO black hole echoes hint at general-relativity breakdown
}

\section{Gravitational-wave data show tentative signs of firewalls or other exotic physics.}

Zeeya Merali

09 December 2016

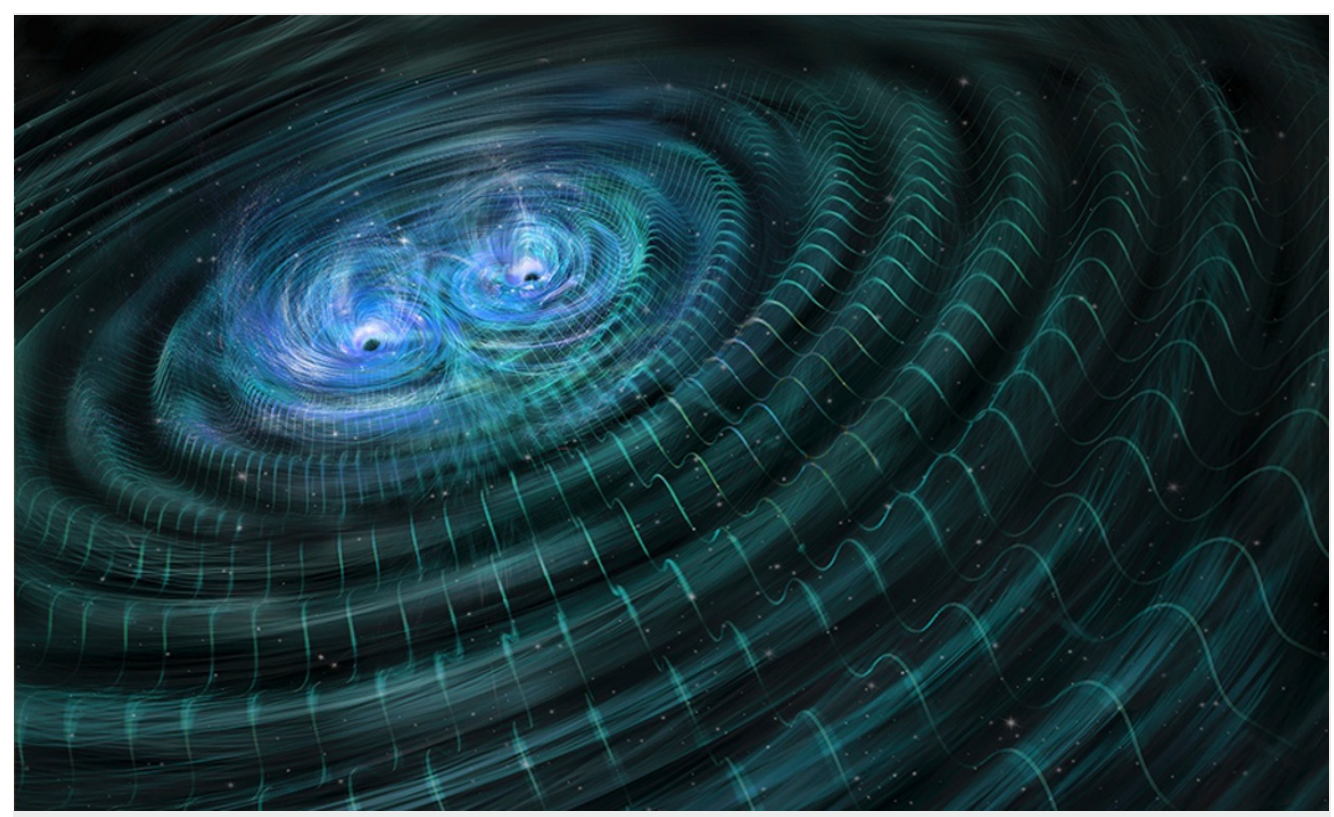

Nicolle R. Fuller/Science Photo Library

Black hole mergers captured by LIGO offer a chance to explore new physics.

It was hailed as an elegant confirmation of Einstein's general theory of relativity — but ironically the discovery of gravitational waves earlier this year could herald the first evidence that the theory breaks down at the edge of black holes. Physicists have analysed the publicly released data from the Laser Interferometer Gravitational-Wave Observatory (LIGO), and claim to have found "echoes" of the waves that seem to contradict general relativity's predictions ${ }^{1}$.

The echoes could yet disappear with more data. If they persist, the finding would be extraordinary. Physicists have predicted that Einstein's hugely successful theory could break down in extreme scenarios, such as at the centre of black holes. The echoes would indicate the even more dramatic possibility that relativity fails at the black hole's edge, far from its core.

If the echoes go away, then general relativity will have withstood a test of its power - previously, it wasn't clear that physicists would be able to test their non-standard predictions.

"The LIGO detections, and the prospect of many more, offer an exciting opportunity to investigate a new physical regime," says Steve Giddings, a black-hole researcher at the University of California, Santa Barbara. The LIGO team says that it is aware of the prediction and searching its data for echoes.

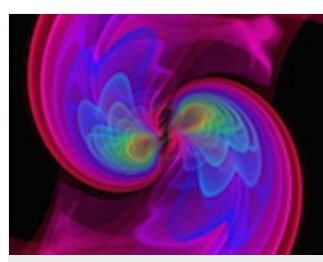

How LIGO forged the path to victory

\section{Beyond reach?}

The edge of a black hole, known as its event horizon, was long thought to lie beyond experimental reach. According to general relativity, anything that crosses the barrier will be captured by the black hole and have no chance of escape. It will be drawn to the black hole's core, where all of the hole's matter is concentrated. "Black holes were thought to be like bottomless pits," says cosmologist Niayesh Afshordi at the University of Waterloo in Canada.

In the standard picture, this leaves nothing at the event horizon, and someone unlucky enough to cross it wouldn't notice any sudden change in the environment. But in 2012, physicists based in California realized that if quantum physics is correct, then the event horizon should be replaced by a firewall, a ring of high-energy particles that would burn any matter that passes through to a crisp and that contradicts general relativity ${ }^{2}$. The alternative is that black holes are firewall-free, but this would imply that quantum theory is wrong. 
Other exotic theories that contradict general relativity also predict some structure at the horizon; for instance, some versions of string theory say that black holes are really 'fuzzballs': tangled up threads of energy with a fuzzy surface in place of a sharply-defined event horizon ${ }^{3}$. However, there did not seem to be any way to peer at the event horizon to find out what, if anything, was there, says Afshordi.

\section{Strange deviations}

That changed in February, when LIGO announced the first direct detection of gravitational waves, or ripples in space-time. The waves were generated when two black holes merged ${ }^{4}$. Soon after, a team of physicists led by Vitor Cardoso at the Superior Technical Institute in Lisbon proposed that if there are any strange deviations from general relativity — such as a firewall — these black-hole mergers would also release a series of echoes after the initial gravitational-wave burst.

The echoes arise because a firewall or any other kind of structure would effectively create a smeared-out region at the traditional event horizon. The inner edge of this region is the conventional event horizon, the boundary beyond which no light particles, or photons, can escape. The outer edge is more porous: a typical photon that crosses this boundary will be trapped by the black hole, but some will be able to escape,

depending on their angle of approach. The effect would also partly trap gravitational waves released by the

black-hole merger. They would bounce back and forth between the inner and outer edge with some escaping each time.

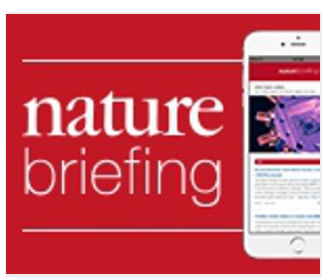

The best science news from across the web, direct to your inbox - free!

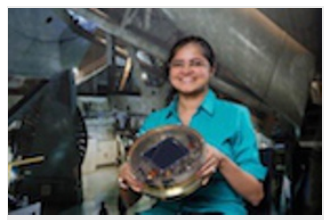

Young scientists poised to ride the gravitational wave

Afshordi's team set up a simple model in which the black hole is surrounded by mirrored walls, rather than just a conventional event horizon, and applied to it properties of each of the three black-hole mergers so far captured by LIGO. This revealed what the precise time interval between repeated echoes should have been if the black holes have any structure at their event horizons - around 0.1 seconds, 0.2 seconds and 0.3 seconds. And when they looked at the LIGO data, they found that the release of gravitational waves in all three mergers was followed by successive echoes at exactly those intervals.

Two Black Holes Merge into One

SXS Project (nww.black-holes.org)

\section{Improved sensitivity}

The echoes could be a statistical fluke, and if random noise is behind the patterns, says Afshordi, then the chance of seeing such echoes is about 1 in 270 , or 2.9 sigma. To be sure that they are not noise, such echoes will have to be spotted in future black-hole mergers. "The good thing is that new LIGO data with improved sensitivity will be coming in, so we should be able to confirm this or rule 
it out within the next two years."

LIGO member Alessandra Buonanno, a physicist at the Max Planck Institute for Gravitational Physics in Potsdam, Germany, says that LIGO scientists are working on an analysis of echo signals.

The simple mirror model used by Afshordi is too crude to say whether firewalls, fuzzballs or something else created the echoes. More-sophisticated models could potentially discriminate between these alternatives, he says, by predicting the amplitudes of the echoes, and how quickly they die out.

But although the team's paper offers "tantalizing hints" of a departure from general relativity, so far these are just hints, says Giddings. And he questions whether Afshordi's mirror model can ever reveal the cause of the deviations from general relativity — in part because the theories that predict them only provide vague descriptions of what replaces the event horizon, making it tough to accurately model them. A "basic problem here is we don't know what is a good physical description of a firewall, or fuzzball".

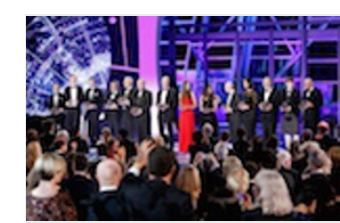

Black-hole fireworks win big in multimillion-dollar science prizes

Nature I doi:10.1038/nature.2016.21135

\section{References}

1. Abedi, J., Dykaar, H. \& Afshordi, N. Preprint at https://arxiv.org/pdf/1612.00266 (2016).

2. Almheiri, A., Marolf, D., Polchinski, J. \& Sully, J. J. High Energy. Phys. 2013, 62 (2013).

3. Mathur, S. D. Fortsch. Phys. 53, 793-827 (2005).

4. Abbott, B. P. et al. Phys. Rev. Lett. 116, 061102 (2016).

5. Cardoso, V., Hopper, S., Macedo, C. F. B., Palenzuela, C. \& Pani, P. Phys. Rev. D 94, 084031 (2016). 